\title{
E-SAÚDE E COMPLEXIDADE: UMA PROPOSTA PARA O DESENHO DE POLÍTICAS PÚBLICAS
}

\author{
E-health and Complexity: A Propose of Public Policy Design
}

\author{
Veronica A. Rojas-Mendizabal'; Arturo Serrano-Santoyo²; Cristián Castillo-Olea³; \\ Amanda Gomez-Gonzalez ; Roberto Conte-Galvan ${ }^{5}$
}

Resumo Objetivos: Neste artigo se apresenta conceito de e-Saúde como sistema sociotécnico. Para isso, considerando sua natureza multifatorial, se adota perspectiva interdisciplinar. Materiais e Métodos: Dada a interferência das Tecnologias da Informação e Comunicação (TIC) no âmbito social e em particular no setor da saúde, sugere-se que a e-Saúde constitua um sistema aberto onde a interação e a interdependência de aspectos tecnológicos, socioculturais, normativos e econômicos cumpram um papel chave na caracterização do sistema, dado que uma aproximação meramente unidisciplinar resultaria insuficiente para a análise geral. Resultados: Uma perspectiva a partir da ciência da complexidade pode extrair novos alcances até o fortalecimento do ecossistema da e-Saúde em níveis local, regional e nacional, aumentando sua efetividade e impactando como importante habilitador de bem-estar social. Conclusão: Argumenta-se que esta perspectiva é particularmente importante na formulação de políticas públicas e em programas de formação de recursos humanos, isso devido à importância do surgimento de novos desafios que a digitalização e a globalização impõem às novas gerações de profissionais do setor da saúde.

Palavras-chave: e-Saúde, Ciência da Complexidade, Inovação, Políticas Públicas, Ecossistemas.

Abstract Aims: This article offers a conceptualization of e-Health as a socio-technical system, in that regard, we adopt a perspective that considers its multifactorial and interdisciplinary nature. Materials and Methods: Given the penetration of Information and Communications Technology (ICT) in the social fabric, particularly in the health sector, we suggest that e-Health constitutes an open system where the interaction and interdependency of technology, sociocultural, regulatory and economic factors play a key role in the characterization of the system, hence, a disciplinary approach is insufficient for its integral analysis. Results: A perspective from complexity science may provide new findings toward the strenghthening of the e-Health ecosystem at local, regional and national levels. Conclusion: We argue that in order to face the challenges of digitization and globalization in the health sector, this perspective is particularly important to supporting the development of public policy strategies and capacity building programs.

Keywords: e-Health, Complexity Science, Innovation, Public Policies, Ecosystem.

\footnotetext{
1. E-mail: vrojas@cicese.edu.mx. Pesquisadora do Centro de pesquisa CICESE. - Mestre e doutoranda em Telecomunicações.; 2. Pesquisador titular do Centro de pesquisa CICESE - Doutor em Telecomunicações; 3. Pesquisadora autônoma em Universidade Autonoma do Baja California. - Mestre em Tecnologia da Informação e doutoranda em Desenvolvimento Global; 4. Gerente de Desenvolvimento Governamental da Agência Espacial Mexicana (AEM) - Doutora em telecomunicações; 5. Pesquisador titular do Centro de pesquisa CICESE - Doutor em telecomunicações. Recebido em 07/04/2014. Aceito, após revisão, em 02/06/2014.
} 


\section{Introdução}

O crescimento dos sistemas de processamento, distribuição e transporte de informação tem tido grande impacto no setor da saúde, criando oportunidades para melhorar serviços, ampliar sua cobertura e buscar enriquecer os processos envolvidos na gestão da informação sanitária, tanto de caráter administrativo como operativo de pacientes e especialistas. Esse crescimento tem sido acompanhado por processos de convergência, onde a digitalização e a globalização são forças que moldam as funções e a estrutura dos ecossistemas da saúde.

A e-Saúde é afetada por elementos socioculturais que cumprem um papel chave na adoção e apropriação da tecnologia no setor saúde. Ao considerar a natureza multidimensional da e-Saúde e a preponderância do usuário final como seu centro de gravidade, permite-se argumentar que, para o entendimento da operação do dito ecossistema de forma integral, uma visão unidisciplinar não é suficiente.

A adoção da convergência tecnológica, ao incentivar a inovação, fortalece a infraestrutura e sobretudo cuida da qualidade e cobertura dos serviços de e-Saúde, para que sejam integrados e acessíveis à população, ao adquirir esse novo enfoque. Isso resultará no aproveitamento do potencial das TIC como veículo de prosperidade social.

A proposta consiste em utilizar um método que permite observar um corte de realidade que se coadune com "guiar" o desenvolvimento e fortalecimento do ecossistema de e-Saúde ou de algum de seus subsistemas, como, por exemplo, o subsistema de e-Saúde/ telediagnóstico, tele-educação em saúde, telemonitoramento, etc. Nesse processo, a intervenção ou o projeto de e-Saúde estará sujeito a interações com agentes internos e externos que dão lugar ao surgimento (emergencial) de propriedades, comportamentos não esperados, processos de auto-organização e retroali- mentação, atributos que denotam sua natureza dinâmica, adaptável e complexa ${ }^{1-4}$.

O objetivo é explorar o ecossistema de e-Saúde pela perspectiva da complexidade e oferecer um marco conceitual para sua abordagem sistemática, com ênfase na formulação e proposta de implementação de políticas públicas. Além disso, pretende-se coadunar o desenvolvimento de políticas públicas, que, por um lado contribuam para um melhor planejamento dos projetos e programas de e-Saúde e, por outro, promovam a criação de contextos em contínua aprendizagem para aprimorar serviços e processos de e-Saúde.

O artigo está organizado da seguinte maneira: na seção 2 se discute o processo de construção da e-Saúde como sistema dinâmico complexo. $\mathrm{Na}$ seção 3 , se descreve brevemente a importância e entendimento dos ecossistemas de e-Saúde. Na seção 4, apresenta-se a proposta de gestão da e-Saúde pela perspectiva da complexidade com fim de contribuir para o desenvolvimento e implementação de políticas públicas que impulsionem seu desenvolvimento e apropriação. Apresenta-se o caso de telediagnóstico como um exemplo básico que permite observar os benefícios de aplicar a perspectiva da complexidade em torno da e-Saúde. Por fim, apresentam-se recomendações e conclusões, enfatizando o caráter exploratório da proposta, reconhecendo o início de uma busca por alcançar, planejar de maneira holística e inclusiva os problemas relativos a projetos de criação e fortalecimento de infraestrutura e processos de e-Saúde, com um enfoque interdisciplinar que atenda às necessidades dos atores do ecossistema com mediação da tecnologia para otimizar suas funções e alcançar seu propósito de bem-estar social.

\section{A Construção do e-Saúde como um Sistema Complexo}

Dados os rápidos avanços tecnológicos e sua convergência associada, argumenta-se que os componentes, 
processos e intervenções de e-saúde não podem ser vistos isoladamente. E-saúde não pode ser conceituada como equipamentos de infraestrutura, sendo esta uma abordagem fragmentada, que limita a sua eficácia e impacto social.

O artigo propõe que a e-Saúde é um sistema aberto, onde a interação e interdependência de fatores tecnológicos, socioculturais e econômicos desempenham papel fundamental na caracterização desse ecossistema. Para levar a cabo nossa análise, voltamo-nos para a ciência da complexidade, que oferece perspectiva que ajuda a entender como os contextos sociais, econômicos e políticos estão interligados. Auxilia para identificar como os sistemas sociotécnicos não são mecânicos, mas também imprevisíveis, com os processos de feedback, interdependência, emergência e auto-organização, que são características da dinâmica de sistemas complexos. Além disso, a perspectiva da complexidade pode extrair novas descobertas para a criação de um quadro, envolvendo os atores mais importantes e diferentes níveis de gestão envolvidos na dinâmica do sistema ${ }^{5,6}$.

Com a introdução da análise da complexidade como proposto e e-saúde como um sistema, conceituamos o pensamento complexo sobre sistema sociotécnico, tal como um corpo coerente de idéias conectadas que nos permitem observar um corte de realidade do trabalho no sistema, que permite, por sua vez, compreender as mudanças e as inter-relações dos subsistemas e atores envolvidos ${ }^{7}$.

Um sistema complexo é difícil de se desintegrar. Suas características coletivas não podem ser fornecidas em sua totalidade, nem seus componentes individuais. Interconectividade é a essência de sistemas complexos; ou seja, esses sistemas compreendem uma rede de nós interligados cujas interações definem sua natureza ${ }^{8}$. Um ambiente é "complicado", quando diferentes subsistemas interagem de tal forma que o total adquire a natureza por si só, realiza adaptações em resposta às mudanças nas condições, onde novas estruturas com novas propriedades surgem como resultado da interação de componentes ${ }^{9}$.

A enumeração dos fatores acima leva nossa análise à proposta do sistema de e-Saúde visto como complexidade assim como estratégia para análise e implementação em diferentes contextos. O comportamento multifatorial da e-Saúde e a interação entre seus componentes apoiam a sua conceituação como um sistema complexo dinâmico e adaptável em que é necessário romper padrões paradigmáticos, que só se concentram no lado tecnológico do sistema ${ }^{10}$.

Na prática, a conjugação das situações acima se aproxima de um acoplamento entre indivíduos e a tecnologia, assim como entre indivíduos, com tecnologia, para produzir a troca de informações, o que, por sua vez, leva a mudanças nos padrões e processos na sociedade e no ecossistema de e-Saúde, a fim de cumprir sua finalidade (ver figura 1).
Ecossistema de e-Saúde

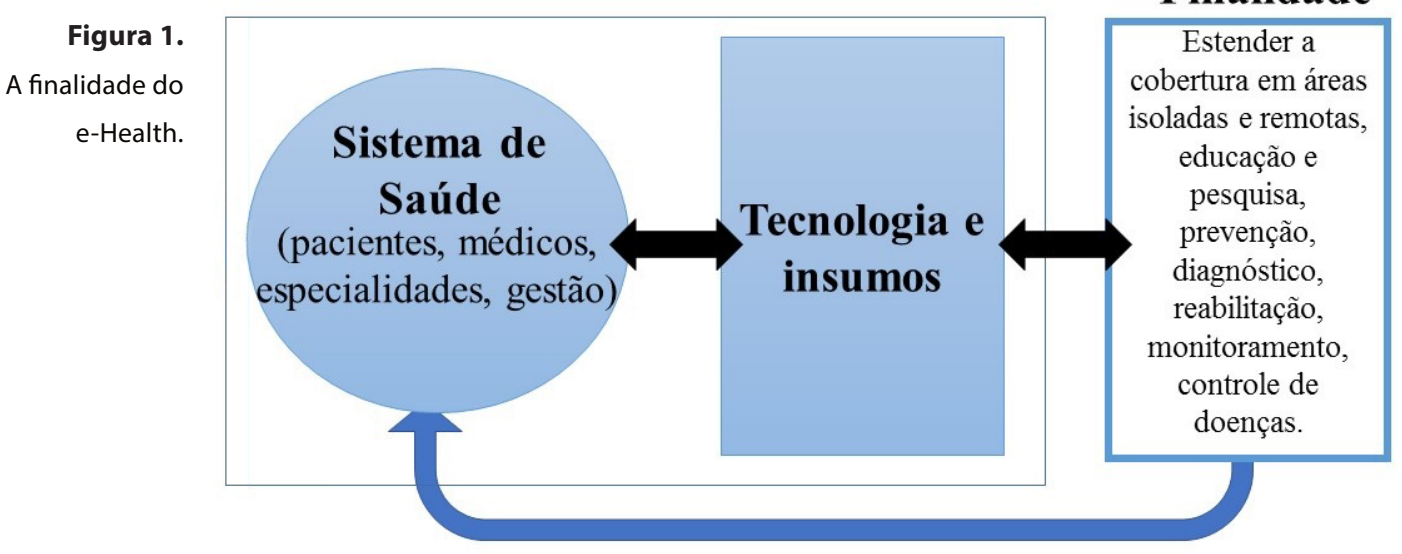


Nesta perspectiva, as interações dos pacientes, médicos especialistas, gestores e tomadores de decisão no ambiente socioeconômico e cultural utilizam a tecnologia como um meio para atingir vários objetivos, tais como: expansão da cobertura em áreas isoladas e remotas, educação e pesquisa, prevenção, diagnóstico, reabilitação, monitoramento etc. ${ }^{11}$.

Os aspectos de convergência e interdisciplinaridade adquirem relevância em torno do trabalho colaborativo que a e-Saúde exige para seu estudo e tratamento abrangente. Em seguida, a proposta é que a abordagem de um problema ou de um conjunto de problemas em e-Saúde seja realizada de maneira interdisciplinar, que envolve um processo de responder a perguntas a fim de resolver um problema, ou como Rolando Garcia coloca: "para pensar de forma diferente sobre o contexto da resolução de um problema". A interdisciplinaridade ocorre na forma como o problema é abordado, e não pelo número ou tipo de disciplinas que os membros de uma equipe de trabalho dominam, ou seja, a coordenação das abordagens disciplinares é necessária para integrá-los em uma abordagem comum ${ }^{6,12}$. A interdisciplinaridade é concebida, para efeitos da presente análise, como um processo, em vez de um produto.

No processo de construção da e-Saúde como sistema complexo, adotamos a proposta de Rolando Garcia ${ }^{6}$ como um marco conceitual e metodológico de trabalho interdisciplinar, que aplicaremos em nosso caso à investigação das interações sociedade-tecnologia.

Propõe-se então uma construção com os seguintes elementos $^{13}$ (figura 2):

a) Gera-se uma visão abrangente do projeto a fim de definir o objetivo do programa ou projeto de e-Saúde a desenvolver.

b) Planejamento de perguntas condutoras.

c) Desenvolvimento de Marco Conceitual. Análise por parte dos participantes no programa de e-Saúde da historicidade e da realidade observada do contexto em que se levará a cabo a intervenção. Isto inclui o entendimento da dinâmica do ecossistema e suas interações.

d) Para chegar a realidade desejada, se adota a estratégia sugerida por Castañares-Maddox ${ }^{5}$ sobre sistemas socioambientais, nos quais se identificam três níveis de gestão na tomada de decisão. Esta estratégia é uma tentaviva de "atuar sobre a complexidade" para conseguir uma conexão entre teoria e prática. Os três níveis são:

- Estratégico

- Tático

- Operativo

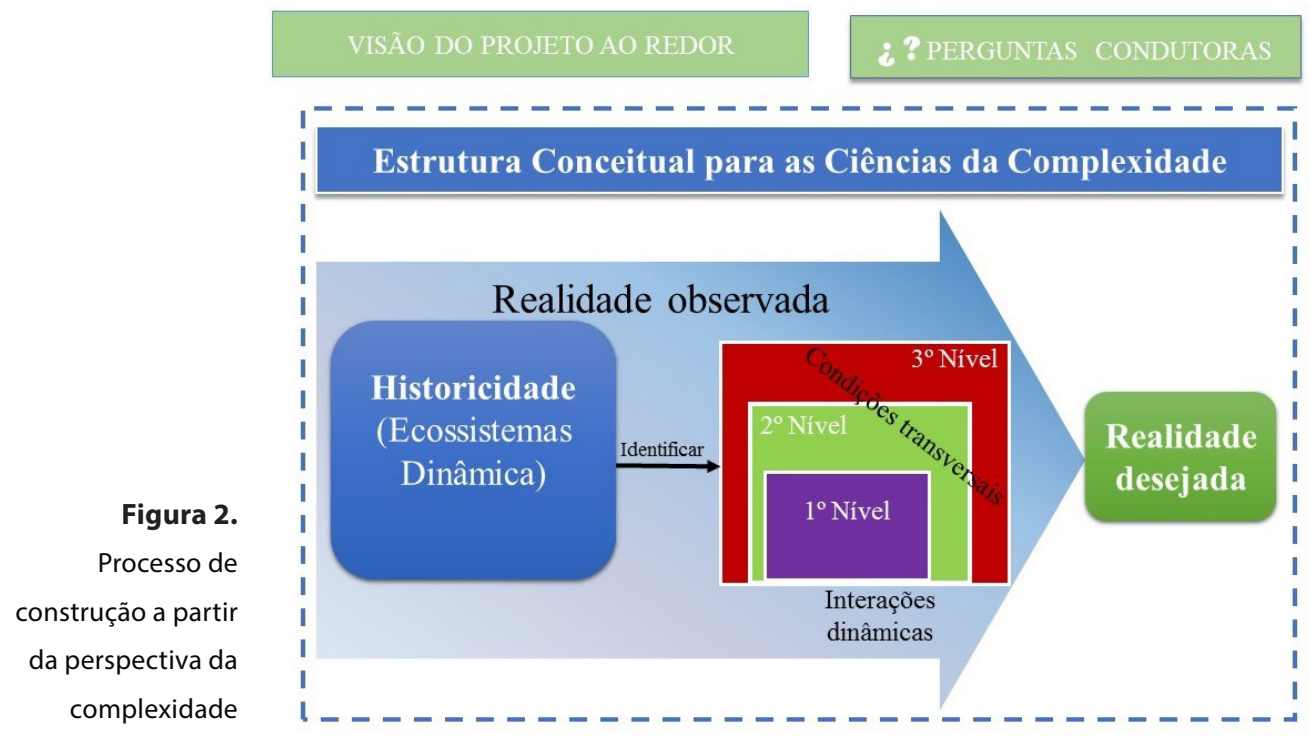


Estes três níveis dão lugar a fluxos de entrada e saída, os quais determinam nas suas inter-relações a estrutura do programa ou projeto visto como sistema complexo. Desta forma, no desenvolvimento do sistema complexo (programa ou projeto de e-Saúde) a tomada de decisões emerge da interação dos agentes nos três níveis dando lugar a ações com grau de coerência e integração.

\section{O Ecossistema de e-Saúde}

Antes de falar sobre um ecossistema de e-saúde, é importante definir os dois conceitos. Para definir e-Saúde é importante distinguir alguns termos que são atualmente amplamente empregados no momento da utilização da tecnologia aplicada à saúde, tais como a Telemedicina e Telessaúde. A Figura 3 mostra a relação entre os termos e-Saúde, Telessaúde e Telemedicina. De acordo com a Organização Mundial de Saúde ${ }^{14}$ Telemedicina é definida como a utilização de telecomunicações para diagnosticar e tratar a doença. Telessaúde é um termo mais amplo, que inclui vigilância, promoção da saúde e funcionamento da saúde pública, bem como a assistência de computadores e telecomunicações para apoiar a gestão, o acompanhamento, a literatura e o acesso ao conhecimento em medicina. E-saúde, é então considerado como a transferência de recursos e cuidados em saúde por meio eletrônico: a entrega de informações de saúde para profissionais de saúde e consumidores, incluindo o uso do comércio eletrônico e negócios eletrônicos na prática de saúde. Consideramos importante que ao definir os termos, se parta de uma base epistemológica para a compreensão do tema por pessoas pertencentes a diferentes áreas do conhecimento. No entanto, reconhece-se que, no cotidiano, os termos são utilizados indistintamente.

O termo ecossistema refere-se a uma comunidade composta por um conjunto de seres vivos interrelacionados e pelo ambiente em que vivem. No caso das telecomunicações, Rehman ${ }^{15}$ define um ecossistema de telecomunicações como a interação sistemática dos seres vivos (Humanos) e não vivos (Tecnologia \& Economia) em um contexto particular. Em todo o ecossistema, o ciclo de vida vai depender da forma como os diferentes subsistemas interagem no contexto.

Rehman ${ }^{16}$ menciona ainda que as causas das falhas nos sistemas de e-Saúde se dão, geralmente, não levando em conta todos os elementos envolvidos. É por isso que esses autores sugerem que é chave realizar uma análise do ecossistema, neste caso, tendo em conta elementos como: as políticas de governança, regulação, modelagem financeira, infraestrutura tecnológica, de serviços e os atores interessados. Com isso, um ecossistema de e-Saúde é capaz de refletir de forma abrangente a situação socioeconômica do lugar em que o projeto ou programa será implementado.

Watts $^{17}$ sugere a complexidade como uma opção relevante para a compreensão dos sistemas de saúde.

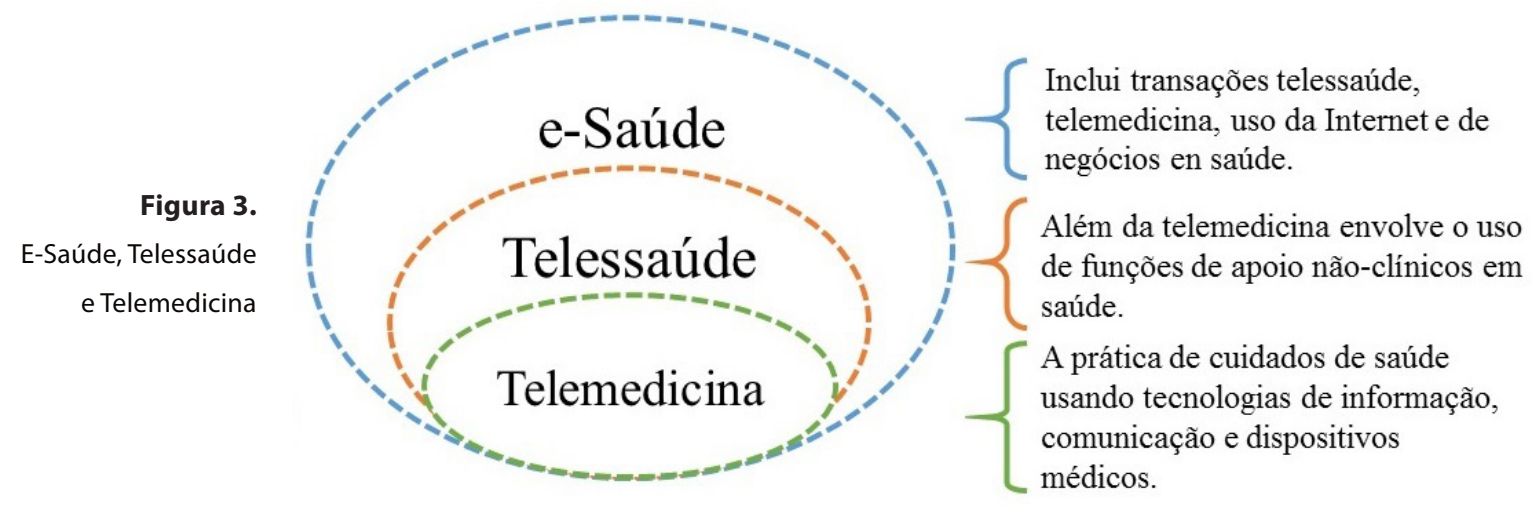


Ou seja, é preciso entender que um ecossistema de saúde está aninhado a outros ecossistemas. Assim, vemos a importância de desenvolver um ecossistema de e-Saúde antes de começar a discutir a implementação de um projeto, porque, como mencionado ${ }^{15}$, usando uma abordagem ecossistêmica correta,será possível determinar a vida útil de um projeto, tentar ser autossustentável e usar diferentes elementos a favor do ser humano. Considera-se, ainda, que o projeto irá evoluir com o tempo, dependendo da eventual mudança no desempenho de seus atores.

A Figura 4 mostra a proposta de ecossistema de e-Saúde, em que se identificam três grandes agentes: humanos, tecnológicos e socioeconômicos ${ }^{18}$. Agentes humanos referem-se a todos os envolvidos no serviço de e-Saúde, quais sejam, médicos, pacientes, gestores, formuladores de políticas, entre outros. Cada um deles desempenha um papel importante no ecossistema, pois, de acordo com as suas capacidades, necessidades e de outros fatores, se estabelece um padrão do que é necessário para o desenvolvimento de um projeto. Agentes tecnológicos referem-se a diferentes dispositivos biomédicos, software para a saúde, os meios de transmissão e armazenamento, entre outros. Estes são os insumos necessários para melhorar o serviço de saúde e são um meio pelo qual podemos dar ferramentas a agentes humanos de maneira a obter e fornecer um serviço de saúde mais eficiente.

Os agentes propostos vão ser influenciados e relacionados por diferentes fatores como Estratégias Investigação + Desenvolvimento + Inovação (I + D + I), fatores humanos, inclusão digital, regulação, patologias do contexto, da cultura e historicidade social, prioridades nacionais, segurança cibernética, construção de habilidades e o grau de desenvolvimento humano do contexto observado. Na próxima seção se abordará as interações dos agentes nos níveis operacional, tático e estratégico para um caso hipotético de telediagnóstico.

Para complementar a análise do ecossistema de telessaúde, deve-se notar o papel fundamental da inovação como estratégia operativa dos projetos em que a informação e o conhecimento se convertem em insumos chaves. Para tal efeito, uma contribuição para o entendimento do conceito de e-saúde/telessaúde está na discussão do papel da pesquisa, trazendo inovação para os serviços de saúde. Há ampla literatura sobre pesquisa translacional. A translação do conhecimento deriva da teoria ator-rede (TAR) de Latour e outros ${ }^{17}$. Pela TAR, traduzir/transladar significa deslocar seres humanos e inanimados, além de objetivos e interesses. Adota-se, para fins desta discussão sobre telessaúde, o conceito $\mathrm{de}^{17}$ pesquisa translacional em saúde como

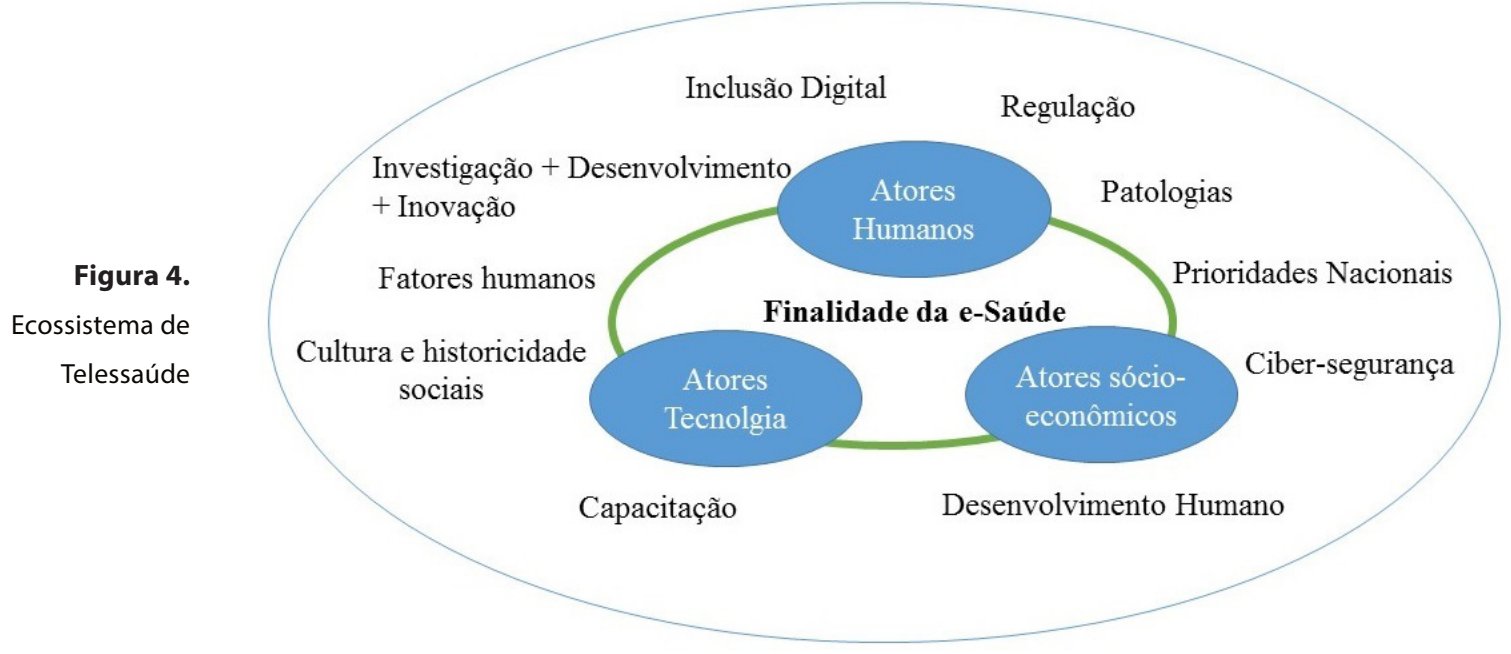


modalidade de pesquisa emergente, com o objetivo principal de diminuir a distância entre a produção do conhecimento nos laboratórios e seu efetivo uso na prática de salvar vidas por meio de intervenções inovadoras para a população. No modelo de Khoury, a pesquisa translacional se divide em 5 etapas no sistema de saúde:

- PTO: começa na descoberta científica através da pesquisa.

- PT1: vai da descoberta até a escolha de sua aplicação.

- PT2: etapa da pesquisa translacional, em que os projetos pilotos localizados acontecem, prevendo a escala no serviço de saúde. Ela compreende a adequação da aplicação candidata até a consolidação da política para sua adoção, ou mesmo a recomendação baseada em evidências;

- PT3: etapa que dissemina efetivamente a política ou recomendação da aplicação prática dos programas no sistema de saúde e seus controles; $e$
- PT4: etapa que aborda a prática da pesquisa translacional e seu impacto na população, envolvendo diretamente as práticas de monitoramento de programas de saúde.

Há ainda uma fase que liga PT4 à PT0, fechando, assim, o ciclo da pesquisa translacional, em que pesquisas fundamentam futuras linhas de pesquisa para o cuidar na saúde (Figura 5).

Ao buscar ampliar o conceito de telessaúde da AMM e OMS, que se resume em TIC para a saúde, chega-se à proposta de ressignificar telessaúde como prática e saber da atenção à saúde que, por mediações tecnológicas, materializa-se no ecossistema de informação e conhecimento em saúde com vários atores-chave ${ }^{19}$. A telessaúde estrutura o processo da pesquisa translacional, pois permite a conexão de pessoas que fazem ciência para salvar vidas nos serviços de saúde. Agregase, assim, essa dimensão ao arcabouço cultural e institucional: a cibercultura em saúde num sistema complexo e autopoiético, que precisa ser desvelado.

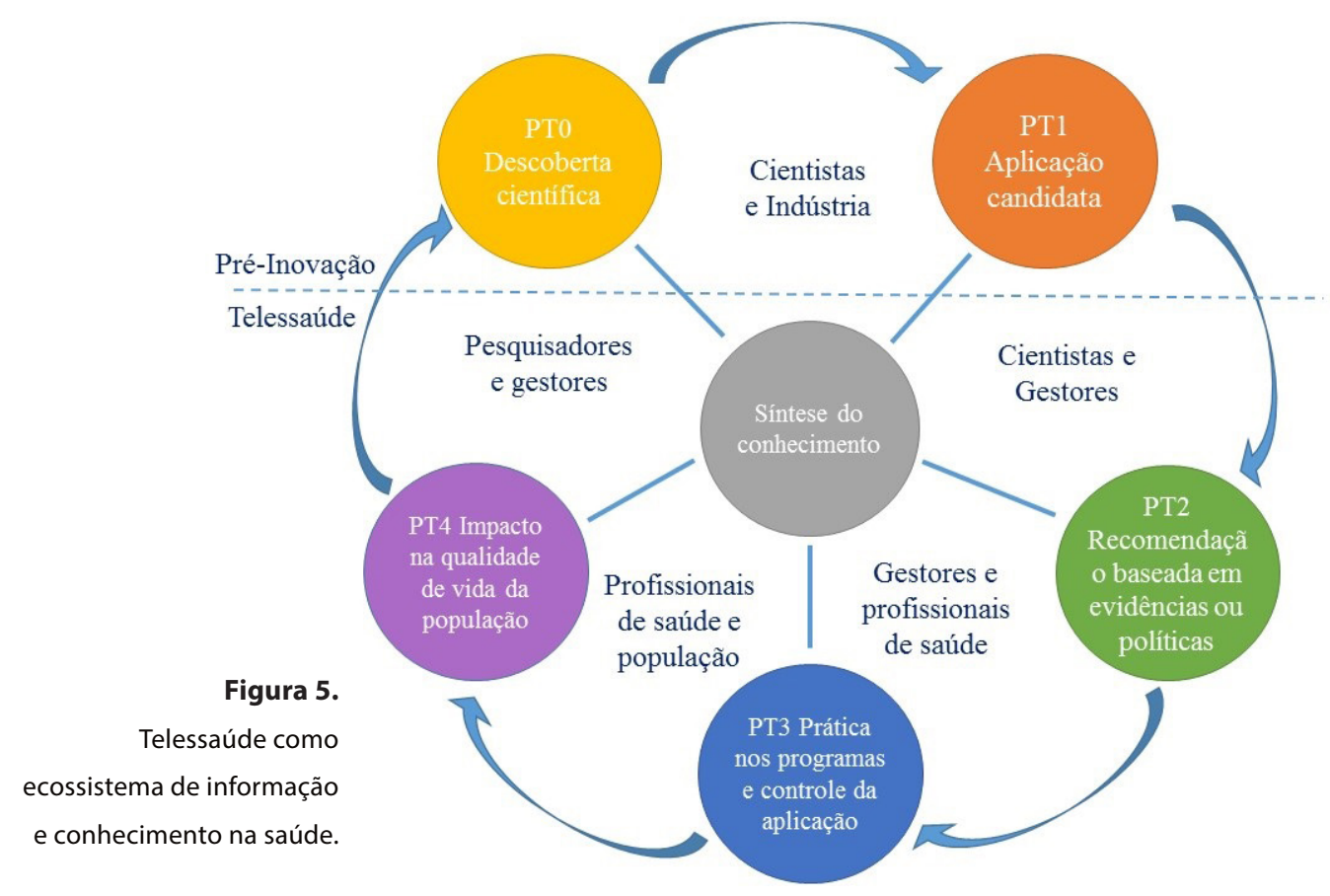




\section{Agindo Sobre a Complexidade: Recomendações para o Desenho de Políticas Públicas em e-Saúde}

Para descrever nossa proposta de desenho de políticas públicas em relação a gestão da e-Saúde na perspectiva da complexidade, em primeira instância, apresentamos a conceituação de diferentes autores sobre políticas públicas. Aguilar Villanueva ${ }^{21}$ considera que as políticas públicas consistem no desenho de uma ação coletiva deliberada, curso que efetivamente toma a ação como resultado de várias decisões e interações e, consequentemente, os fatos reais que a ação coletiva produz. Então, uma política pública é uma solução que é oferecida com base em um status social.

O mesmo autor ${ }^{22}$, menciona que há uma diferença entre políticas de governo e políticas públicas, no sentido de que as últimas não são apenas decisão do ator governamental, mas que constituem um curso de ação que se desencadeia e efetivamente é desenvolvido, intencional e reativamente por vários atores governamentais, por outros poderes do estado, atores políticos, grupos econômicos ou mesmo organizações sociais.

Políticas públicas exigem o consenso social. Portanto para atingir a sua aceitação e implementação, devem ser propostas e discutidas publicamente e principalmente serem convincentes de que solucionarão de forma viável a condição social em pauta. Assim, ao estarem em acordo quanto às soluções propostas para uma determinada condição ou demanda social, os atores sociais podem então ser persuadidos a participar na implementação dessas políticas públicas. Um elemento importante a considerar é que apresentar uma proposta de política pública pressupõe a presença de cidadãos informados e interessados no tópico de debate, cidadãos aos quais devem ser fornecidos espaços para participação ad-hoc ideal, para que tenham efetiva intervenção no processo.
Portanto, segundo o enfoque de sistemas complexos, pode se afirmar que governar orientado por políticas públicas significa governar incorporando a opinião e participação dos cidadãos; o que em verdade constitui o caminho ótimo para gerar políticas públicas ${ }^{23}$.

A Organização Mundial de Saúde afirma que:

Em programas nacionais de saúde, cerca de dois terços dos países não têm uma política nacional de tecnologias de saúde que orientam o planejamento, avaliação, aquisição e gerenciamento de dispositivos médicos. Assim, a ausência de uma política pode ocasionar que sejam feitos investimentos em dispositivos médicos que não reflitam as necessidades prioritárias da população, que são incompatíveis com a infraestrutura e os serviços existentes ou cuja manutenção é muito cara. Estes desperdícios podem minar os sistemas de saúde como um todo, desviando fundos que seriam necessários para outros serviços essenciais ${ }^{24}$.

Introduzir uma análise das políticas públicas de saúde no contexto da complexidade, conforme descrito anteriormente, consiste em identificar e propor uma pergunta condutora. Então, é necessário definir uma intervenção específica e o contexto, bem como, definir seus objetivos e escopo. Por exemplo, no caso de telediagnóstico, a questão principal é relacionar as necessidades e características do contexto, assim como sua historicidade. Ou seja, é necessário efetuar análise da realidade observada no cenário do telediagnóstico para posteriormente intervir no centro de saúde específico.

Neste exemplo de telediagnóstico, em que um centro remoto localizado em uma área rural requer o apoio de um centro especializado para o diagnóstico de uma doença ou patologia específica, perguntas condutoras poderiam ser:

- São relevantes as atuais políticas para melhorar a condição de saúde do contexto rural onde se está intervindo? 
- Como políticas públicas aplicadas a telediagnóstico podem melhorar a cobertura e a qualidade dos serviços de saúde no contexto descrito?

- Como usar o telediagnóstico em contexto específico a fim de melhorar a cobertura e a qualidade dos serviços de saúde?

- Como o telediagnóstico reduz o custo de transporte de pacientes para hospitais especializados?

- Como o telediagnóstico melhora os fatores de custo-benefício e custo-eficiência dos serviços de saúde em uma comunidade rural específica?

- Como o telediagnóstico melhora o índice de desenvolvimento humano (IDH) da população rural e ao mesmo tempo da região ou do país?

- Quais são as qualificações mínimas em uso de meios digitais para aplicação de telediagnóstico em uma população rural?

- Quais são os elementos necessários para a adoção e aplicação de telediagnóstico em uma população rural?

Dependendo do problema a ser resolvido, a pergunta condutora é definida. Se procede à análise dos níveis, como proposto na Figura 6, com a qual temos um recorte da realidade observada particular do contexto. Então, se pode construir coletivamente um sistema integrado de telediagnóstico de modo a identificar as funções e responsabilidades das partes interessadas, inter e intra níveis de atuação.

Nossa análise, para um caso hipotético de telediagnóstico em México, considera a identificação dos agentes que formariam o sistema em três níveis de interação. Para descrever a contribuição deste artigo de forma mais específica, podemos considerar o caso de uma população rural no México que requer suporte de telediagnóstico.

Em nível estratégico, são nacionalmente (para citar os atores principais): A Secretaria de Saúde (SS), o Centro Nacional de Excelência Tecnológica em Saúde (CENETEC) ${ }^{25}$; e internacionalmente: A Organização Mundial de Saúde (OMS); A Organização Pan Americana de Saúde (OPS). Em nível tático: um centro rural de primeiro nível onde a intervenção será executada com a base em um centro especializado. No nível operacional são identificados:o subsistema humano, com médicos especialistas (ME), médicos generalistas (MG) e pacientes (P); o subsistema tecnológico: dotado de dispositivos médicos (DM) e software aplicado à saúde (SWS); e o
Figura 6.

Contextualização preliminar do espaço do estúdio dentro dos três níveis de sistemas complexos no campo da decisão. Casos no México Telediagnóstico

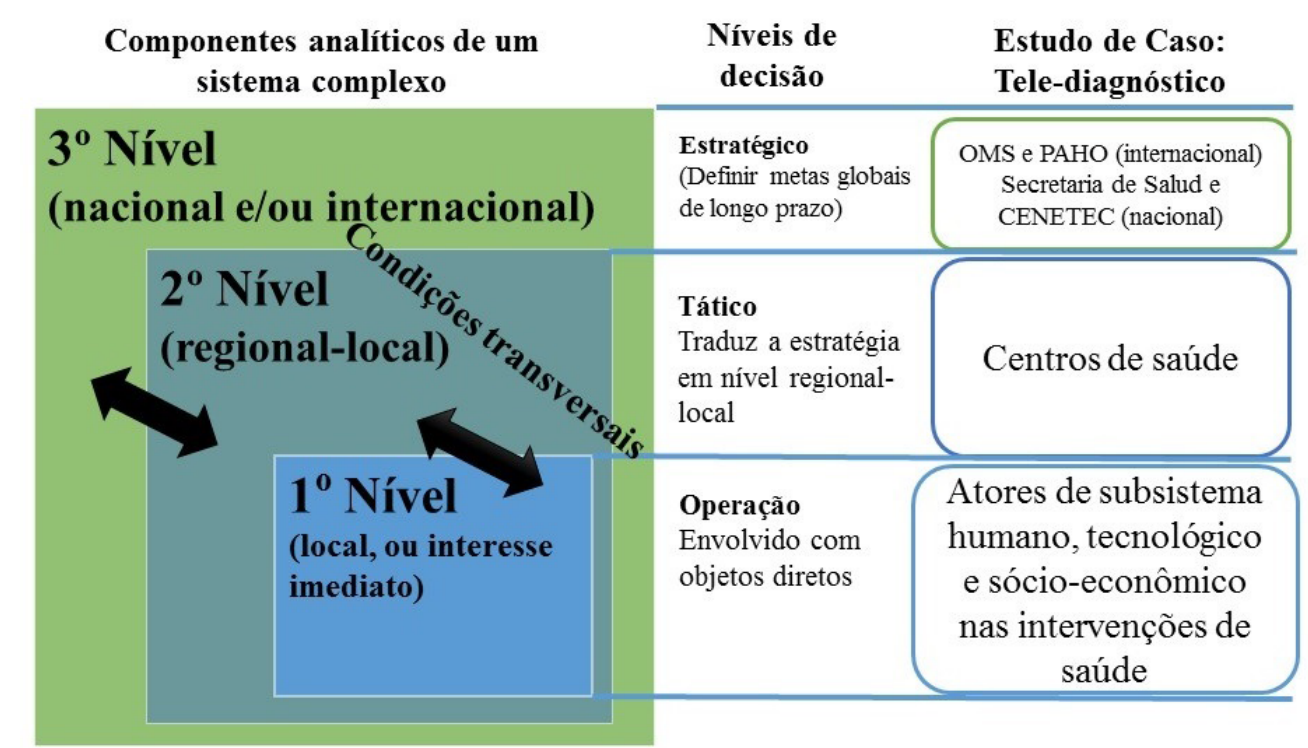


subsistema socioeconômico: infraestrutura física (IF) (acessibilidade, ergonomia e organização de dispositivos e equipamentos), o custo dos fatores envolvidos na intervenção $(\mathrm{Cl})$ (entradas, homem-horas, conectividade etc).

A Figura 7 mostra a interação entre os atores determinados anteriormente na conceituação de nosso espaço de estudo, dentro e entre cada um dos níveis para o exemplo referenciado nesse trabalho. A figura tem como objetivo analisar quais são as interações com mais peso e influência sobre o desempenho e as regras de comportamento de cada ator dentro do sistema para alcançar a realidade desejada na qual se identificariam as interações prioritárias e fluxos que fornecem diretrizes para políticas públicas emergentes em nível operacional.

Para analisar as interações em nível operacional, no caso de telediagnóstico podemos ver que há uma interação entre o clínico geral e o especialista através de dispositivos médicos, tipos diferentes de software aplicado à videoconferência e uma rede de internet. Uma vez que a tecnologia será o fator mediador para que se obtenha o diagnóstico oportuno, de modo a auxiliar na decisão da melhor atuação dos médicos em relação ao paciente. A este respeito, as competências digitais dos médicos são muito importantes. Desta forma, se deve analisar cada uma das interações no âmbito do nível considerado.

Como demonstra a Figura 7, ao analisar o nível tático, este vai possibilitar a mediação entre os níveis operativo e estratégico, por isso, o nível tático desempenha um papel importante no momento da criação de políticas públicas. No exemplo exposto, há interação entre os centros de saúde que estão envolvidos no nível operacional. Também se observa que os custos de insumos (CI) e a infraestrutura física (IF) estão relacionados com cada um destes centros de saúde, uma vez que sua administração depende dos recursos financeiros disponíveis. Como no nível anterior, é necessário realizar uma análise mais detalhada, o que exige um processo de observação em cenário mais específico.

Para o nível estratégico, CENETEC é uma instituição com responsabilidades no processo de e-Saúde, a qual depende da Secretaria de Saúde, de modo que ambas as instâncias interajam diretamente durante todo o processo. Como se pode observar em seguida, a análise das interações inter e intraníveis fornece informações
Figura 7.

Caso exemplificado por telediagnóstico no México.

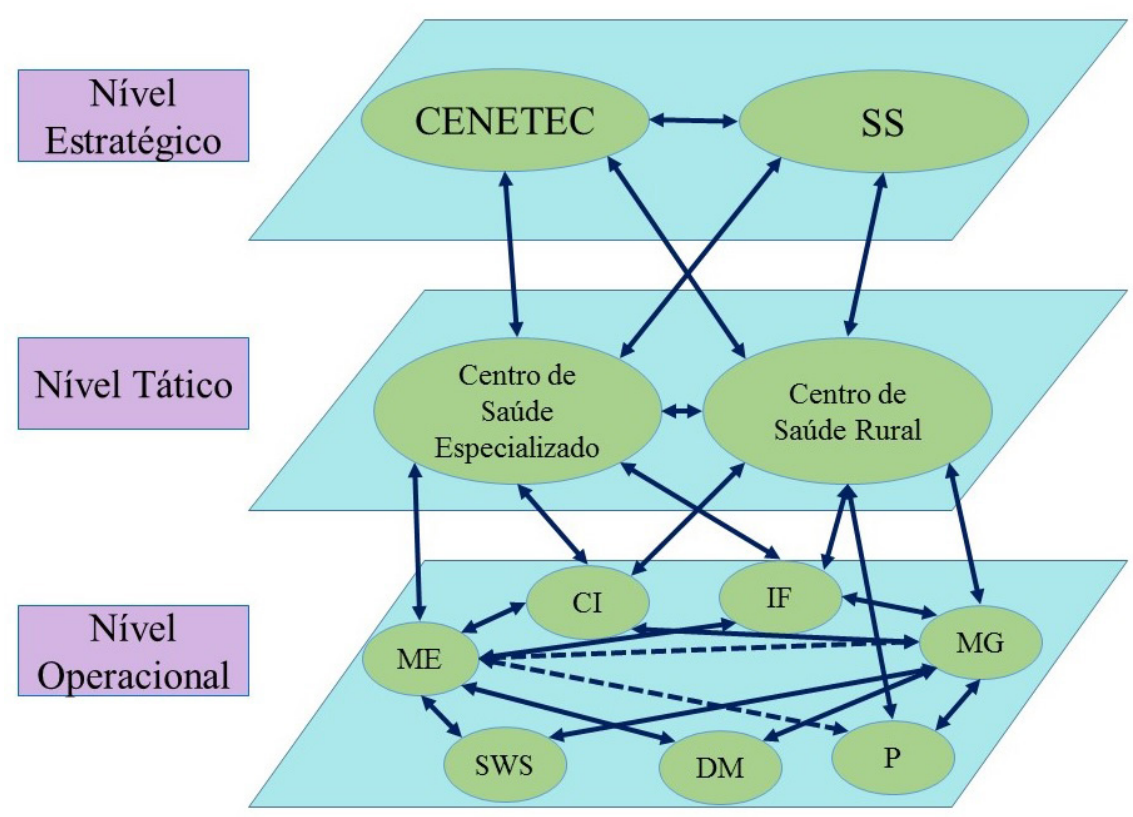


importantes para chegar à realidade desejada, a qual representa um corte da operação do subsistema de telediagnóstico pelo qual se pode planejar melhor a intervenção e por sua vez, dependendo da pergunta condutora, ajudar a definir a política pública associada, que em suma é o objetivo da contribuição proposta no artigo.

A partir da realidade observada é possível sugerir estratégias de implementação e um plano de ação que se encontre em constante observação pelos agentes dos níveis táticos e estratégicos para, por um lado, conceber novas políticas públicas, ou se for o caso, reforçar as já existentes.

Devido à natureza dinâmica e adaptável do ecossistema da e-Saúde, onde o telediagnóstico é realizado, é necessário um processo de feedback entre os diferentes níveis, para otimizar e atualizar periodicamente as políticas associadas. Uma possível análise pode ser realizada usando modelos baseados em agentes (MBA) com o qual você pode gerar diferentes cenários que permitem desenvolver um projeto apropriado da intervenção em si, e que, ao mesmo tempo, suporte o desenvolvimento de políticas públicas, como é mostrado na Figura 8.

Os resultados do modelo baseado em agentes devem ser validados a fim de entregar intervenções práticas que forneçam informações, ligando teoria e prática de proprietários de processos de telediagnóstico e outros processos do ecossistema de e-saúde.

\section{Comentários Finais e Conclusões}

O marco conceitual fornecido nesse conceituação de Telessaúde sugere uma conexão que envolve vários sistemas inter-relacionados (cognitivos, tecnológicos, linguísticos, socioculturais e outros), assim suporta uma abordagem interdisciplinar como uma "ponte conceitual" entre disciplinas que pode, a princípio, ser vista como incompatível.

Reconhece-se que a fragmentação das disciplinas relacionadas com processos de troca e fluxo de informações entre os atores-chave da e-saúde é um fator importante como parte constituinte do ecossistema. No entanto, argumenta-se que a fragmentação excessiva e a falta de uma visão clara do e-saúde a serviço da sociedade pode não permitir uma aproximação a uma realidade nos dispositivos envolvidos, equipamentos, processos, programas e seres humanos, estes últimos os mais importantes.

A título de fechamento e como futura análise, identificamos desafios importantes para a implementação de projetos de e-Saúde:

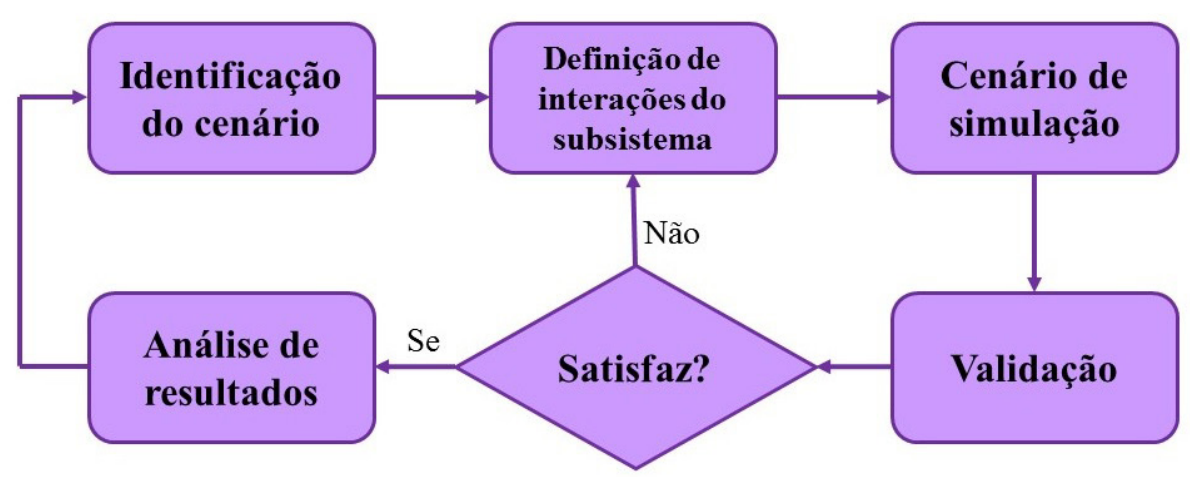

Figura 8. Modelagem de Processo $(\mathrm{MBA})^{26}$ 
A possibilidade de integração de uma abordagem epistemológica sólida para cada programa e projeto de e-Saúde capaz de interpretar e explicar a interação entre sistemas sociais e tecnológicos. Com isso, representar um marco teórico para a abordagem da e-Saúde, como alternativa para a análise e operação, que podem, eventualmente, contribuir para a concepção de projetos (inclusão digital do setor saúde, de dispositivos médicos e redes de próxima geração e outros), programas de educação de forma holística e contribuir para a sua aplicação para o bem estar social.

\section{Referências}

1. Anderson RA. Case Study Research: The View From Complexity Science. Qualitative Health Research. 2005 May 1;15(5):669-85.

2. Almeida-Filho N. Complejidad y Transdisciplinariedad en el Campo de la Salud Colectiva: Evaluación de Conceptos y Aplicaciones. Salud Colectiva. 2006 May;2(2):123-46.

3. Ramis Andalia RM, Sotolongo Codina PL. Aportes del pensamiento y las ciencias de la Complejidad al estudio de los determinantes de la salud. Revista Cubana de Salud Pública. 2009 Dec;35(4):65-77.

4. Organisation for Economic Co-operation and Development (OECD) Global Science Forum. Applications of Complexity Science for Public Policy: New Tools for Finding Unanticipated Consequences and Unrealized Opportunities. Erice, Sicily: OCDE; 2009 Sep.

5. Castañares Maddox EJ. Sistemas complejos y gestión ambiental: el caso de Corredor Biológico Mesoamericano México. Comisión Nacional para el Conocimiento y Uso de la Biodiversidad. México, DF;

6. García, R. Sistemas Complejos. Gedisa; 2006.

7. Morin E, Pakman M. Introducción al pensamiento complejo. Barcelona: Gedisa; 1994.

8. Liu Y-Y, Slotine J-J, Barabasi A-L. Observability of complex systems. Proceedings of the National Academy of Sciences. 2013 Jan 28;110(7):2460-5.

9. Axelrod RM, Cohen MD. Harnessing complexity: organizational implications of a scientific frontier. New York: Basic Books; 2000.

10. Kilkki K. Quality of Experience in Communications Ecosystem. Journal of Universal Computer Science. 2008;14(5).
11. Congreso de Colombia. Ley No 1419 "Lineamientos para el desarrollo de la Telesalud en Colombia" [Internet]. 2010. Available from: http://wsp.presidencia.gov. co/Normativa/Leyes/Documents/ley141913122010.pdf

12. Abrahamsson, P S O., Ronkainen, J. \& W J. Agile software development methods: Review and analysis. VTT Publications. 2002;478:98.

13. Aguilar de la Peña JM. Pobreza y Marginación en la Mixteca Poblana: un análisis desde el enfoque de Sistemas Complejos. In: Ortiz Espejel B, Duval G, editors. 1. ed. Puebla, Pue: Universidad Iberoamericana Puebla : Colegio de Posgraduados Puebla : Secretaría del Medio Ambiente y Recursos Naturales, Puebla; 2008.

14. World Health Organization. Trade, foreign policy, diplomacy and health [Internet]. World Health Organization. 2014 [cited 2014 Mar 2]. Available from: http://www.who. int/trade/glossary/story021/en/

15. Rehman Laghari K, Connelly K. Toward total quality of experience: A QoE model in a communication ecosystem. IEEE Communications Magazine. 2012 Apr;50(4):58-65.

16. Shehadi R, Tohme W, Bitar J, Kutty S. Anatomy of an E-Health Ecosystem. Booz \& Company Inc.; 2011.

17. Watts DJ. Six degrees: the science of a connected age. New York: Norton; 2003.

18. Rojas-Mendizabal VA, Serrano-Santoyo A, Conte-Galvan R, Gomez-Gonzalez A. Toward a Model for Quality of Experience and Quality of Service in e-health Ecosystems. Procedia Technology. 2013 Jan;9:968-74.

19. Carroll M, James JA, Lardiere MR, Proser M, Rhee K, Sayre MH, et al. Innovation Networks for Improving Access and Quality Across the Healthcare Ecosystem. Telemedicine and e-Health. 2010 Feb;16(1):107-11.

20. Silva AB, Morel CM, Moraes IHS de. Proposta conceitual de telessaúde no modelo da pesquisa translacional. Revista de Saúde Pública. 2014 Apr;48(2):347-56.

21. Aguilar LF. La implementación de las políticas. México, D.F.: Miguel Ángel Porrúa; 1996.

22. Aguilar LF. La hechura de las políticas. México, D.F.: Miguel Ángel Porrúa; 1996.

23. Lahera E. Introducción a las políticas públicas. Santiago, Chile: Fondo de Cultura Económica; 2002

24. Organización Mundial e la Salud. Dispositivos médicos [Internet]. Organización Mundial de la Salud. 2010 [cited 2014 Apr 2]. Available from: http://www.who.int/ mediacentre/factsheets/fs346/es/

25. Centro Nacional de Excelencia Tecnológica en Salud. Atribuciones de CENETEC [Internet]. CENETEC. 2012 [cited 2014 Apr 2]. Available from: http://www.cenetec. salud.gob.mx/interior/atribuciones.html

26. He S. Agent-based modelling using MATLAB. Available from: http://www.cs.bham. ac.uk/ Szh/teaching/matlabmodeling/Lecture14_body_Agent.pdf 